\title{
A simplified controller and detailed dynamics of constant off-time peak current control
}

\author{
Alex Van den Bossche*, Ekaterina Dimitrova**, \\ Vencislav Valchev ${ }^{* *}$, Firgan Feradov ${ }^{* *}$
}

\begin{abstract}
A fast and reliable current control is often the base of power electronic converters. The traditional constant frequency peak control is unstable above $50 \%$ duty ratio. In contrast, the constant off-time peak current control (COTCC) is unconditionally stable and fast, so it is worth analyzing it. Another feature of the COTCC is that one can combine a current control together with a current protection. The time dynamics show a zero-transient response, even when the inductor changes in a wide range. It can also be modeled as a special transfer function for all frequencies. The article shows also that it can be implemented in a simple analog circuit using a wide temperature range IC, such as the LM2903, which is compatible with PV conversion and automotive temperature range. Experiments are done using a $3 \mathrm{~kW}$ step-up converter. A drawback is still that the principle does not easily fit in usual digital controllers up to now.
\end{abstract}

K e y w or ds: analog processing circuits, circuit analysis, circuit simulation and modelling, current control, PV conversion

\section{Introduction}

The advantage of using a peak current control is that one can combine a protection and a control in the same item. The constant frequency peak current control is even more widely used, but, without tricks, it is typically unstable beyond $50 \%$ duty ratio. This occurs in fly-back step down and step-up converters [1].

The literature proposes a lot of complex variants of combined current control. A Constant Frequency/Constant Off-Time Hybrid pulse width modulation (PWM) control of digitally controlled boost converter with PID auto-tuning without current sensing is proposed in [2]. A PWM technique with constant on/off-time control for a synchronous buck dc/dc converter in order to reduce the switching frequency and switching losses is presented in [3]. A hybrid fixed frequency current controller suitable for DCDC Applications that uses both the turn-on and the turn-off current mode and the sliding mode control techniques is presented in [4]. A power controller for dealing with load uncertainties in domestic induction-heating applications is proposed in [5]. It combines different variable frequency PWM strategies - constant duty cycle, constant on-time, and constant off-time modulations for reaching the target power level while minimizing the switching frequency. Applications of current mode constant off time control are also presented in a current-fed full-bridge boost $\mathrm{DC} / \mathrm{AC} / \mathrm{DC}$ converters [6]. A comparison of current controls is presented in [7] and [8]. The fundamental difference between constant on-time control and constant-frequency peak-current-mode is analyzed through a proposed model in [7]. In [8] authors compare four current mode control schemes. A burst-mode control for the LLC resonant converter to improve the light-load efficiency is presented in [9].

The pure constant off time control has also a lot of applications in this era. It is quite popular in step down converters for LED lamp control, like PAM99700 universal high brightness LED driver, HV9910B universal high brightness LED driver, IS31LT3918 high voltage LED driver with switch dimming, SN3912 universal high brightness LED driver. In [10], a control of LLC resonant converters for LED PWM dimming is presented. The LED intensity is controlled by the ratio between the ontime and off-time of the PWM dimming signal. Constant off time current control is also applied in low power stepup controllers like LTC3813 - $100 \mathrm{~V}$ current mode synchronous step-up controller; LTC3814-5 a current mode synchronous step-up Controller. Also applications in motor control are possible, for example in brushless DC motors control (BLDC) [11].

In this article, we look at a simple and low cost and low power consumption variant. The aim is to return to basics and to look carefully at simple schemes and basic transfer functions. The typical applications in mind are Photovoltaic (PV) boost converters, battery charging, power factor controllers, BLDC and Switched reluctance motor control. The wide tolerance of changes in inductance is also safe if the inductor saturates and would get a much lower value. Even in the presence of severe disturbances, the frequency never goes to infinite. This is in contrast with hysteresis control which can destroy the

*Department of Electrical Energy, Systems and Automation, Ghent University, Technologiepark 913, B-9052 Zwijnaarde, Gent, Belgium, alex.vandenbossche@ugent.be, ** Department of Electronics and microelectronics, Technical University Varna, Studentska 1, Varna 9010, Bulgaria, edimitrowa55@gmail.com, vencivalchev@hotmail.com** 
converter due to disturbances on the current measurement. The designer has also a typical problem that the level of the current protection should be higher than the controlled range but not too high. All these things can be combined in one single device using a COTCC.

\section{Principle}

First we remember the basic principle. The transistor is turned on, when the current is higher than the peak current set value $I_{L I M I T}$, the transistor is turned off during some fixed time, see Fig. 1(a). If the current remains too high, the transistor is kept off. This means that one needs a kind of retriggerable monostable multivibrator. The principle is given in Fig. 1(b), but the practical implementation of it will be given in Fig. 8(a).
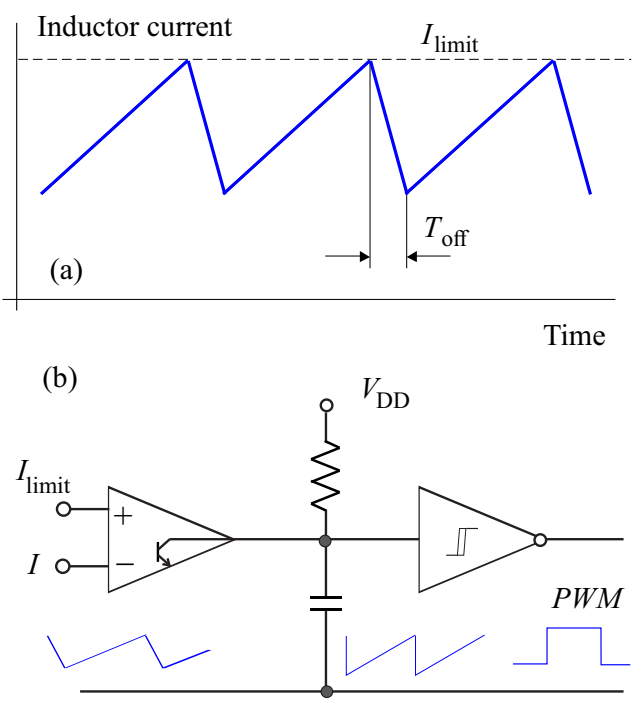

Fig. 1. (a) - constant off time peak current control, (b) - implementation using a simple controller

\section{Mathematical model of the ideal case}

For large signal models, one can better refer to simulation. For small signals, the system can be considered linear for small changes. In the case of COTCC, one considers that the change is small so that the frequency does not change much. When a step is applied, the duty ratio changes for one period, to get again at the original value after the step.

\subsection{Time domain approach}

Qualitatively, if the step can occur at any moment of the period, the chance to have a peak current increases proportional with the elapsed time up to $100 \%$ after one period. Or one could say that statistically the average delay is $50 \%$ of a switching period, shown in Fig. 2. This is very fast for a current control, which would normally need already a current feedback loop, and its dynamics, if a normal voltage PWM method was used.

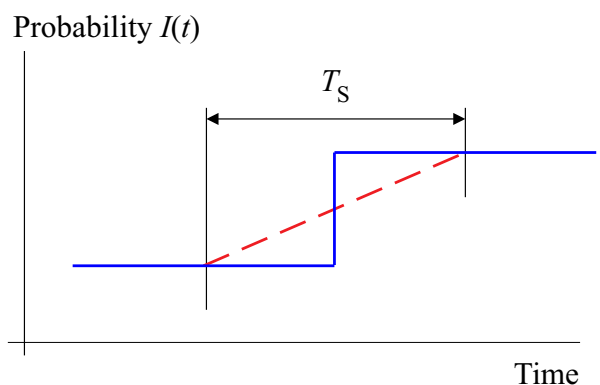

Fig. 2. Step response if the ripple is ignored in a probability approach

\subsection{Transfer function analyzer approach}

Laplace, and $Z$-transform are quite risky in PWM type of environments to be applied without any trouble. However, one can sample a cosine wave and multiply the result of it afterwards with sine and cosine to get the amplitude and angle of the applied component in the sampled wave. This is in the same way that a transfer function analyzer does, and it is the basic of Fourier transfer analysis, Fig. 3.

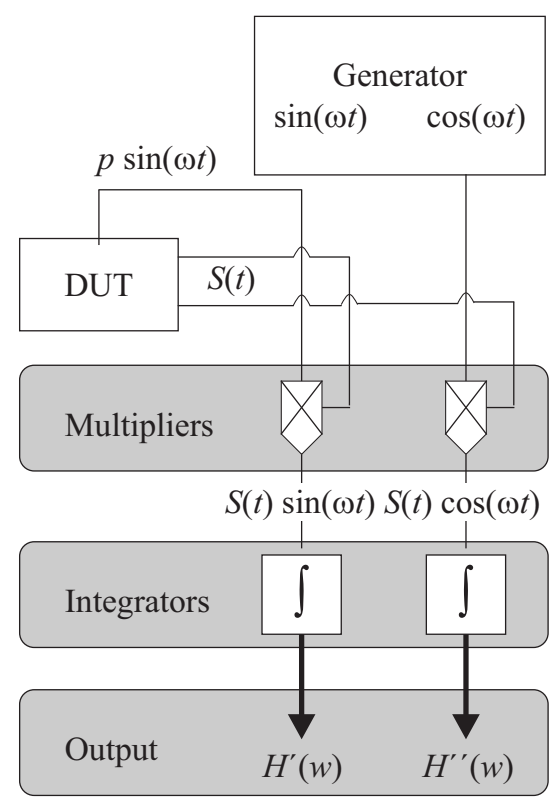

Fig. 3. Transfer function analysis schematic (modified from Barsaukov \& MacDonald 2005)

The peak current control has in fact a sampling action at each period, which is represented in Fig. 4. If one does it for different sampling cosinewave/sampling frequency ratio $k v$, obtains the graph of Fig. 5 .

Note that the transfer function follows a sine wave dependence for all frequencies, except for odd multiples of half of the switching frequency. The function $r v(k v)$ fits well the frequency dependence observed in Fig. 5. The following equation could be seen as a fitting function

$$
r v(k v)=\frac{\sin \left(2 \pi \frac{k v}{2}\right)}{2 \pi \frac{k v}{2}}
$$




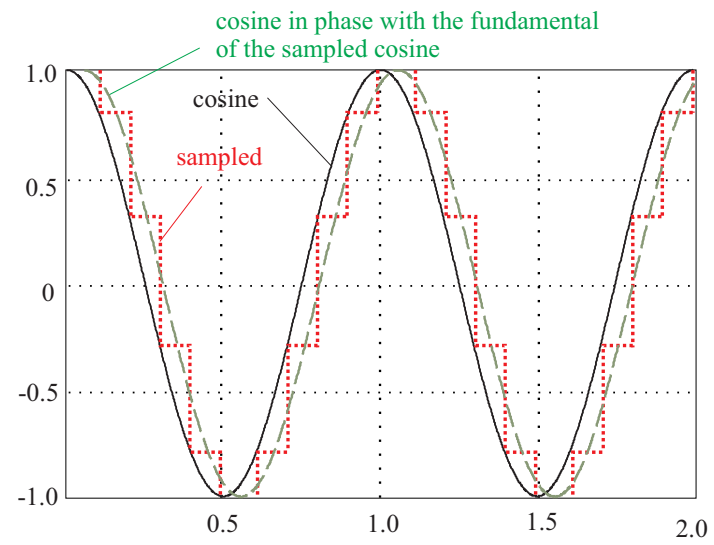

Fig. 4. Cosine function, the sampled cosine, and a cosine in phase with the fundamental of the sampled cosine

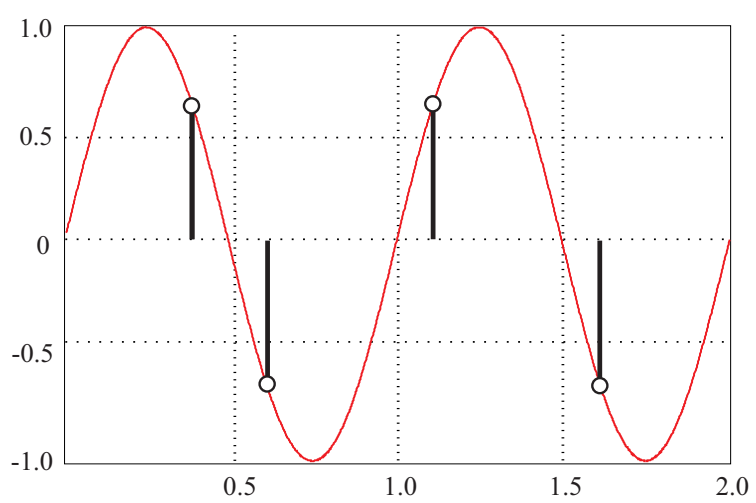

Fig. 6. Sampling at with an applied sine of half the switching (sampling) frequency

Note that the result at $k v=0.5,1.5,2.5,3.5$ doubles compared to the simple expression (1). At those frequencies, the amplitude and phase are dependent on the relative phase of the signals, which is not shown in Fig. 5. hence, could be seen as some worst case. Practical engineers often experience that oscillations at half of the switching frequency occurs in a lot of fast controlled PWM systems. It is also happening in constant frequency peak current control at about $50 \%$ duty ratio.

\subsection{Frequency domain transfer function}

If one uses the Laplace transform, he has the problem that several step inputs (at a different time) result in the same output, so the system is not time independent, which is a requirement to have a Laplace transform. However, in the case that only a sinusoidal perturbation is applied, one can look at the components of the same frequency in phase with that sine and quadrature in the output. This is known as a Fourier transfer function. Nevertheless one can try modeling the sampling phenomenon, where the change of the output current is sampled and hold during one period at each turn-off.

This result in the transfer function of a sample and hold is, a Dirac is converted into a pulse

$$
F_{s h}=\frac{1-e^{-s T_{s}}}{s T_{s}} .
$$

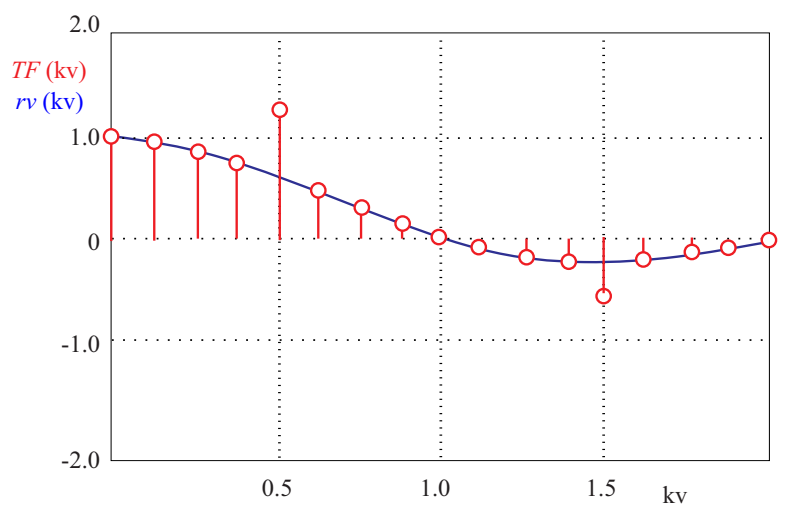

Fig. 5. Transfer function amplitude $r v(k v)$ as function of sample frequency/sine frequency ratio $k v$

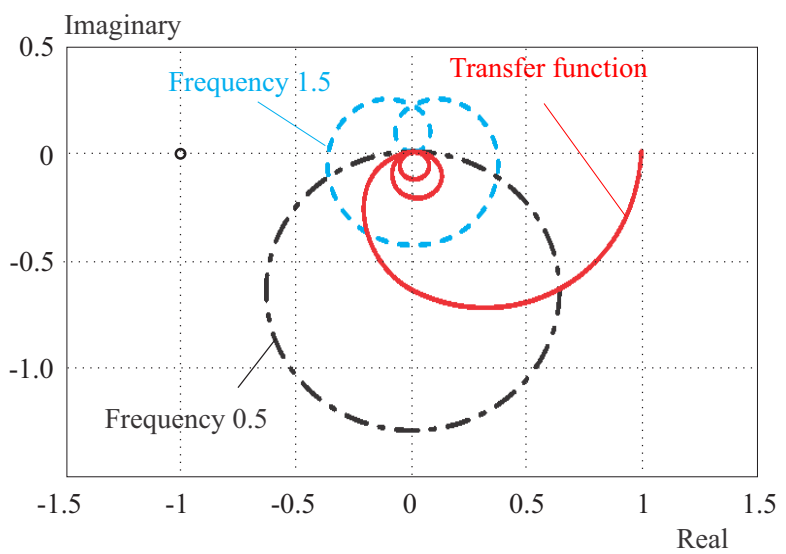

Fig. 7. Nyquist diagram of the transfer function, including the frequencies 0.5 and 1.5

This can be written as a delay and an amplitude effect, such as in communication theory. This is also, with replacement of $s$ by $j \omega$, for Fourier

$$
F_{1}(\omega)=e^{\frac{-j \omega T_{s}}{2}} \frac{\sin \frac{\omega T_{s}}{2}}{\frac{\omega T_{s}}{2}} .
$$

Note that, in the communication theory, one often does not care much about the average delay of $T_{s} / 2$ in the first factor, but in control it is very important to take it into account.

The last part results in the typical $\operatorname{sinc}(x)$ function in telecommunication [12]. The normalized definition is

$$
\operatorname{sinc}(x)=\frac{\sin (\pi x)}{\pi x} .
$$

Another remark is that, for half of the switching frequency, $\omega=2 \pi T_{s} / 2$, the result is depending on the phase which is applied as shown in Fig. 6.

Sampling at half of the switching frequency and also at odd multiples of half of the switching frequency generates a phase and amplitude change, depending on the relative phase of the applied signal. It also generates a maximal component which can be twice as big as $F_{l}(\omega)$ would 

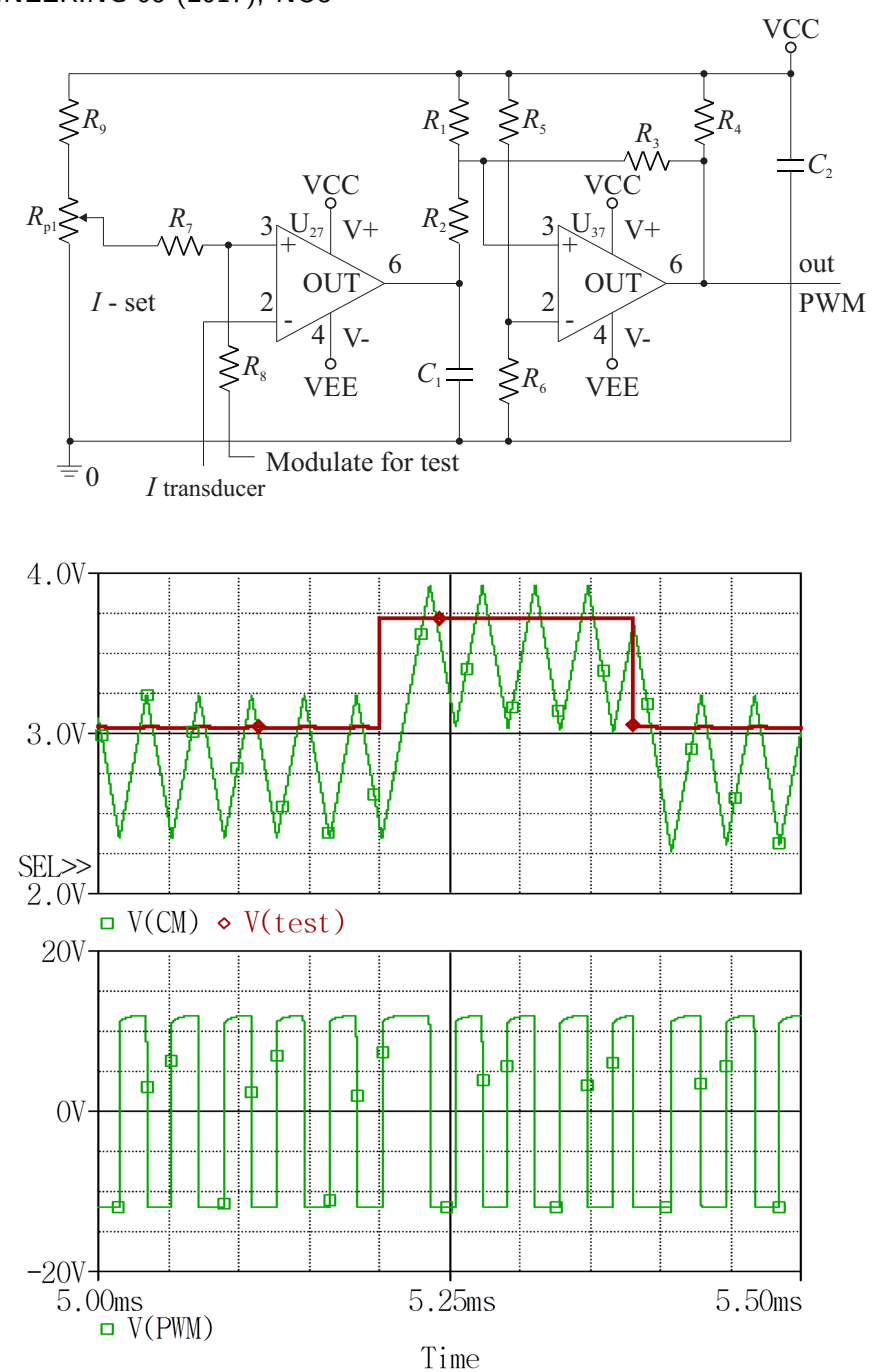

Fig. 8. The scheme in real, as well for simulation, corresponding to $14 \mu$ s off time (up), Step response of the current control (down)
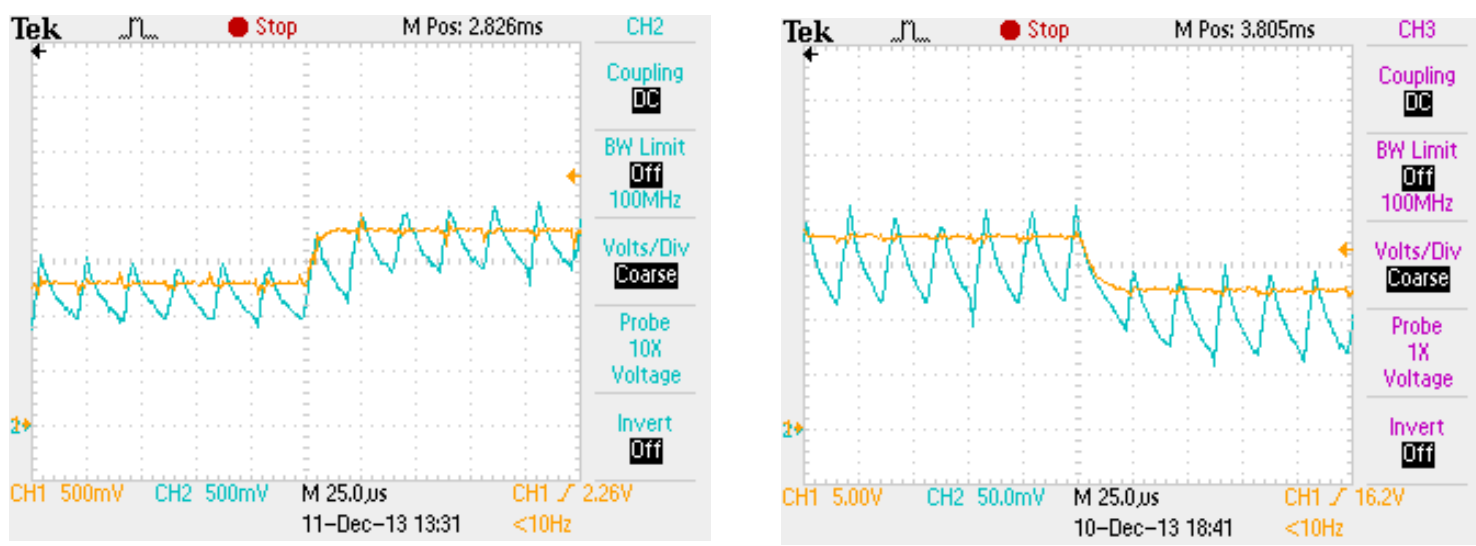

Fig. 9. The set value of the peak current and the inductor current in a step-up converter: (a) - rising edge, (b) - falling edge

suggest. The fundamental reason is that the component of the mirror frequency adds to the normal frequency.

One can take this in account and have a multiplying factor specifically at odd multiples of half of the switching frequency

$$
A(\zeta)=2 \cos (\zeta) e^{(-j \zeta \omega T)}, \quad-\pi / 2<\zeta<\pi / 2 .
$$

Using this correction, we get also compatible with the transfer analyzer method of Fig. 7. The phase change and the bigger amplitude at half of the switching frequency may particularly be risky in feedback, which tends to result in instabilities at that frequency if some phase delay is combined. In general, a stability theory should be valid for all applied frequencies, also for input frequencies higher than the switching frequency. This is the case here. So it seems that we should also pay attention in resonances in the load which are at 1.5 of the switching 
frequency (or even above). The amplitude of the transfer function is three times lower, but all phase angles appear. So, at that frequency, a phase shift in control does not really help to stabilize.

\section{Simulation of the proposed practical circuit}

\subsection{Practical implementation of the circuit}

The circuit, Fig. 8(a), is simple enough to get an accurate simulation in PSpice. The simulation, Fig. 8(b), shows that some $4.2 \mu \mathrm{s}$ time is needed before the ideal transistor is turned off. This is mainly due to the internal delay of the used integrated circuit.

$$
\begin{aligned}
& \mathrm{R} 1=10 \mathrm{k}, \mathrm{R} 2=2.2 \mathrm{k}, \mathrm{R} 3=47 \mathrm{k}, \mathrm{R} 4=10 \mathrm{k}, \\
& \mathrm{R} 5=22 \mathrm{k}, \mathrm{R} 6=22 \mathrm{k}, \mathrm{R} 7=10 \mathrm{k}, \mathrm{R} 8=100 \mathrm{k}, \\
& \mathrm{R} 9=10 \mathrm{k}, \mathrm{R} 1=10 \mathrm{k}, \mathrm{C} 1=0.47 \mathrm{nF}, \mathrm{C} 2=100 \mathrm{nF}
\end{aligned}
$$

The impulse response is shown on Fig. 8(b), where $\mathrm{V}(\mathrm{CM})$ is the output voltage of the current to voltage transducer, $\mathrm{V}$ (test) is the modulated input for test reference voltage, and $\mathrm{V}(\mathrm{PWM})$ is the $\mathrm{PWM}$ signal in closed loop Boost DC-DC converter. The circuit operation is verified by this simulation.

\section{Performance of the circuit}

In the real circuit also, a delay depending of the level shifters is introduced and a delay due to the current transducers. A series of practical measurements have been conducted using a test setup of a boost dc-dc converter. The tests are conducted with input voltage of $100 \mathrm{~V}$ DC. Some of the parameters of the test inverter are as follows: the used inductor has $600 \mathrm{H}$ inductance, the load is $60.9 \Omega$ in parallel to $20 \mu \mathrm{F}$. The current of the inductor was used as an input for the current control.

In Fig. 9, the behavior of the current control is shown. The graphs depict the voltage output of the current transducer and the step of the reference voltage. As it can be seen, the control manages to follow the changes in the reference voltage without any delays or fluctuations.

By means of experiment the inductor has been reduced to $10 \mu \mathrm{H}$, it results in much more ripple, but it is still not unstable.

\section{Features and drawbacks}

\subsection{Dynamics}

The circuit shows a dead-beat control dynamic, even if the inductor is changed in a wide range. This is rather exceptional in control theory. There is some steady state error, but that can be corrected by adding the ripple to the set value of the current.
In the continuous mode the difference between peak and average current is

$$
\begin{aligned}
I_{\text {peak }}-I_{\text {av }} & =\frac{T_{\text {off }}}{2 L} \Delta V, \\
\text { where } \quad \Delta V & =\left(V_{\text {out }}-V_{\text {in }}\right)
\end{aligned}
$$

In discontinuous mode and the off time gets bigger than the free wheel period, hence, the diode conduction time $\left(T_{\mathrm{d}}\right)$ is

$$
T_{\text {on }}=\frac{I_{\text {peak }} L}{V_{\text {in }}}, \quad T_{\mathrm{d}}=T_{\text {on }} \frac{V_{\text {in }}}{\Delta V} .
$$

After some calculations, one can get

$$
I_{\mathrm{av}}=\frac{I_{\mathrm{peak}}}{2} \frac{\frac{V_{\mathrm{out}}}{\Delta V}}{1+\frac{T_{\mathrm{off}}}{T_{\mathrm{on}}}}
$$

Then correction from peak to average is $I_{\text {peak }}-I_{\mathrm{av}}$.

The correcting value can be shown for the example for $100 \mathrm{~V}$ input and $200 \mathrm{~V}$ output, for the given inductance and off time in Fig. 10. In this way, one can compensate for the steady state error by feed forward.

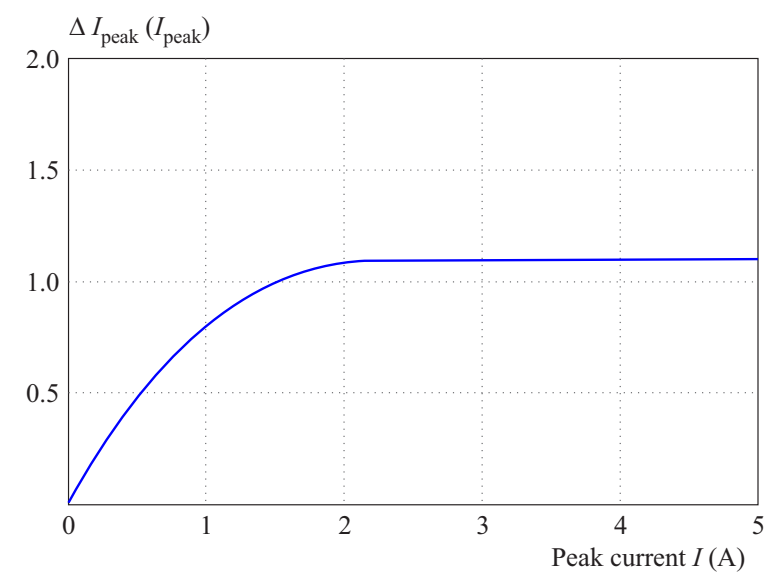

Fig. 10. Steady-state error compensation

\subsection{No limit cycle at duty close to duty ratio $=1$}

Normal fixed frequency PWM does have problems with extreme duty ratios. For example $95 \%$ and $100 \%$ are possible but not in between, as the drivers cannot follow very short pulses. In a current control this results in an instability in that region.

The constant off time control does not show this problem and can go to $100 \%$ duty ratio in a seamless way.

\subsection{Disturbance sensitivity}

If there is a disturbance during the off time, nothing special happens, the transistor remains off or remains somewhat longer off. This disturbance may be caused by the turn off of the transistor, it has the tendency to give a second off time, so getting in a safe condition, until the designer can fix the signals that they get a better quality. This is in contrast with hysteresis control where the frequency can tend to infinite if the disturbance is bigger than the hysteresis band and can destroy the converter even at the first tests. 


\section{Conclusion}

A constant off-time current control has an excellent dynamic behavior in the time domain, which is close to a dead-beat control within one switching period. In the frequency domain, the transfer function can be approximated in a transfer function with a delay of half of the switching period and some $\sin \left(\omega T_{s}\right) /\left(\omega T_{s}\right)$ - type of function for the amplitude. At the half of the switching frequency, and odd multiples of it, a double amplitude occurs, and large phase shifts are observed. This is indeed the Nyquist frequency where the signal frequency and the mirror frequency interfere and add together. There is a steady state error, which can be corrected in feed forward. The constant off time control is not so easy to be implemented in processor circuits, but can be easily made with two low cost comparators integrating timing and Schmitt-trigger action in a small scheme. The experiments and Pspice simulation correspond well and do not change the practical behavior, except for some propagation delay time.

\section{Acknowledgements}

The carried-out research is realized in the frames of the project "Model based design of power electronic devices with guaranteed parameters", DN07/06/15.12.2016 funded by Bulgarian National Scientific Fund.

\section{REFERENCES}

[1] S. S. Ang, "Control Schemes of switching Converters" Power Switching Converters, 3rd ed., CRC Press, Boca Raton FL, 2010.

[2] Y. Na, M. Zhonghan and Hao, "A Multimode Digitally Controlled Boost Converter With PID Autotuning and Constant Frequency/Constant Off Time Hybrid PWM Control" IEEE Trans. Power Electronics, vol. 26, no. 9, Sep 2011, pp. 2588-2598.

[3] Y. Chia-An and L. Yen-Shin, "Digital Pulsewidth Modulation Technique for a Synchronous Buck DC/DC Converter to Reduce Switching Frequency" IEEE Trans. Industrial Electronics, vol. 59, no. 1, Jan 2012, pp. 550-561.

[4] A. Lachichi, S. Pierfederici, J. P. Martin and B. Davat, "Study of a Hybrid Fixed Frequency Current Controller Suitable for DC-DC Applications" IEEE Trans. Power Electronics, vol. 23, no. 3, May 2008, pp. 1437-1448.

[5] A. Dominguez, L. A. Barragan, A. Otin, D. Navarro and D. Puyal, "Inverse-Based Power Control Domestic Induction-Heating Applications" IEEE Trans. Industrial Electronics, vol. 61, no. 5, May 2014, pp. 2612-2621.

[6] S. Jalbrzykowski and T. Citko, "Current-Fed Resonant FullBridge Boost DC/AC/DC Converter" IEEE Trans. Industrial Electronics, vol. 55, no. 3, March 2008, pp. 1198-1205.

[7] L. Jian and F. C. Lee, "New Modeling Approach and Equivalent Circuit Representation for Current-Mode Control" IEEE Trans. Power Electronics, vol. 25, no. 5, May 2010, pp. 1218-1230.

[8] Y. Yingyi, F. C, P. Lee and Mattavelli, "Comparison of Small Signal Characteristics Current Mode Control Schemes for Point of-Load Buck Converter Applications" IEEE Trans. Power Electronics, vol. 28 , no. 7 , July 2013 , pp. 3405-3414.
[9] F. Weiyi, F. C. Lee and P. Mattavelli, "Optimal Trajectory Control of Burst Mode for LLC Resonant Converter" IEEE Trans. Power Electronics, vol. 28 , no. 1, 2013, pp. 457- 466.

10] F. Weiy, F. C. Lee and P. Mattavelli, "Optimal Trajectory Control of LLC Resonant Converters for LED PWM Dimming" IEEE Trans. Power Electronics, vol. 29, no. 2, Feb 2014, pp. 979-987.

11] A. Van denBossche, D. Bozalakov, T. Vyncke and V. Valchev, "Programmable logic device based brushless DC motor control" 14th European Conference on Power Electronics and Applications (EPE 2011), pp. 1-10.

12] F. W. J. Olver, D. M. Lozier, M. Daniel, R. F. Boisvert, C. W. Clark, eds. "Numerical methods" NIST Handbook of Mathematical Functions, Cambridge University Press 2010.

Received 7 March 2017

Alex P. M. Van den Bossche became Member of IEEE in 1993 and a Senior Member (SM) in 2003. He received the $\mathrm{MS}$ and the $\mathrm{PhD}$ degrees from Ghent University, Belgium in 1980 and 1990 respectively. He works and teaches there at the Electrical Energy Laboratory. His research is in the field of electrical drives, power electronics on various converter types and passive components. Since 1993, he is a professor at the same university in the same field. He is co-author of a book: A. Van den Bossche, V. C. Valchev, Inductors and transformers for power electronics. 2005, CRC - press, Boca Raton, Florida, US).

Ekaterina N. Dimitrova became Member of IEEE in 2013. She received the MS degree in Electronics and MS degree in Applied Mathematics from the Technical University of Sofia, Bulgaria in 1978 and 1983 respectively, and the $\mathrm{PhD}$ degree from the Technical University of Varna, Bulgaria in 2012. Since 1989 she has been with the Department of Electronics, Technical University of Varna. She teaches Analysis and Synthesis of Electronic Circuits, Analog Circuits and Microelectronics. Assoc. prof. Dimitrovas research interests include: CAD systems in Electronics, Diagnosis of Analog and Mixed Electronic Circuits, Circuit Analysis and Synthesis and she has published more than 43 papers.

Vencislav C. Valchev was born in Vidin, Bulgaria in 1962. He has become a member of IEEE since 2004. He received the MSc and $\mathrm{PhD}$ degrees in Electrical Engineering from the Technical University of Varna, Bulgaria in 1987 and 2000, respectively. Since 1988 he has been with the Department of Electronics and Microelectronics, Technical University of Varna. His research interests include power electronics, soft switching converters, resonant converters, magnetic components for power electronics, renewable energy conversion. He has published more than 100 papers and a scientific book: A. Van den Bossche, V. C. Valchev, Inductors and transformers for power electronics. 2005, CRC - press, Boca Raton, Florida, US). Prof. Valchev is a member of Science and Research Union in Bulgaria.

Firgan N. Feradov was born in Varna, Bulgaria in 1989. He received Bachelor degree in Electronics and Master degree from Technical University of Varna in 2012 and 2014 respectively. He has participated in Erasmus student mobility programme and worked in Ghent University, Belgium. He is now a PhD student at the Technical University of Varna. 